\title{
Contraception, pregnancy, and induced abortion within 24 months of delivery in eastern, central, and western regions of China: a retrospective cohort study
}

Yan $\mathrm{Che}^{1}$, Yuyan $\mathrm{Li}^{2}$, Xiangying $\mathrm{Gu}^{3}$, Lifang Jiang ${ }^{4}$, Yuanzhong Zhou ${ }^{5}$, Xiaoyu $\mathrm{Hu}^{6}$, Li Jiang $^{7}$, Tongying Cheng ${ }^{8}$, Dong Xiao Jing ${ }^{9}$, Xiaochen Huang ${ }^{10}$, Yongfeng Luo ${ }^{11}$, Wen Lv ${ }^{12}$, Guangli Qiao ${ }^{13}$, Jiandong Song ${ }^{14}$, Wei Xia ${ }^{15}$, Linai Zhang ${ }^{16}$, Yanfei Zhou ${ }^{17}$, and Yan Zhang ${ }^{2}$

${ }^{1}$ Affiliation not available

${ }^{2}$ 1. NHC Key Lab. of Reproduction Regulation(Shanghai Institute of Planned Parenthood Research), School of Public Health, Fudan University

${ }^{3}$ Tianjin Medical University General Hospital

${ }^{4}$ Henan Key Laboratory of Population Defects Prevention, Henan Provincial Research

Institute for Population and Family Planning;

${ }^{5}$ School of Public Health, Zunyi Medical University

${ }^{6}$ Shanghai Women's and Children Health Center

${ }^{7}$ Maternal and Child Health Hospital of Guangxi Zhuang Autonomous Region

${ }^{8}$ Xinjiang Urumqi Maternal and Child Health Hospital

${ }^{9}$ The second affiliated hospital of Chongqing Medical University

${ }^{10}$ Fujian Maternity and Children Health Hospital

${ }^{11}$ Jilin Guojian maternity hospital

${ }^{12}$ Tongde Hospital of Zhejiang Province

${ }^{13}$ Health and Reproductive Health Technical Guidance Service Center of Ningxia Hui Autonomous Region

${ }^{14}$ Affiliated Hospital of Inner Mongolia Medical University

${ }^{15}$ Institute of Reproductive Health, Tongji Medical College, Huazhong University of Science and Technology

${ }^{16}$ Shanxi Maternal and Child Health Hospital

${ }^{17}$ Changsha Hospital for Maternal and Child Health Care,

August 22, 2020

\begin{abstract}
Objective To explore the prevalence of contraceptive use, unintended pregnancy, and induced abortions within 24 months postpartum in eastern, central, and western regions of China and in China overall. Design A retrospective cohort study. Setting Hospital-based Maternity units. Population Women who delivered a live baby between 12 and 24 months. Methods: We randomly selected postpartum women at 60 hospitals in eastern, central, and western regions of China. We applied descriptive statistics and life-table analyses. Main outcome measures Prevalence of contraception, unintended pregnancy, and abortions. Results: A total of 18,045 participants were enrolled and interviewed, and 12-, and 24-month contraceptive rates were $85.7 \%$ (95\% CI 85.2-86.2), and 86.8\% (95\% CI 86.3-87.2), respectively. Condoms accounted for $79 \%$ of contraceptive initiators. The $12-$, and 24 -month rates were $5.3 \%$ (95\% CI 5.0-5.6), and 13.6\% (95\% CI 12.2-15.0) for unintended pregnancy; and $4.0 \%$ (95\%
\end{abstract}


CI 3.7-4.3), and 10.8\% (95\% CI 9.5-12.1) for induced abortion, respectively. Three-quarters of unintended pregnancies ended in abortion. Postpartum contraceptive use was higher in the western region relative to the eastern or central regions, but the risks of unintended pregnancy and induced abortion were also higher in the west. Conclusions: Postpartum contraception use was relatively high in China but dominated by less-effective methods, and these may contribute to higher risks of unintended pregnancy and induced abortion during the postpartum period. Use of long-acting reversible contraceptives and effective and reliable short-acting methods should thus be fostered in postpartum family planning services in China.

\section{Funding}

This work was supported by Science and Technology Climbing Funds from the Shanghai Institute of Planned Parenthood Research (grant no. PD2017-10)

Tweetable abstract Postpartum contraception was dominated by less-effective methods, which may contribute to higher risks of unintended pregnancy and induced abortion during the postpartum period in China.

\section{Introduction}

A short interpregnancy interval increases the risks of maternal and infant mortality and morbidity. Early studies have shown that closely spaced pregnancies are associated with adverse maternal and infant outcomes. ${ }^{(1,2)}$ Family planning can prevent more than $30 \%$ of maternal deaths and $10 \%$ of child mortality when couples space their pregnancies 2-3 or more years apart. ${ }^{(3)}$ In 2013 , WHO recognized postpartum family planning (PPFP) as one of the top priorities with respect to global reproductive health issues. ${ }^{(4)}$

Postpartum contraceptive use varies among countries. A previous study showed that $72 \%$ of women used a contraceptive method by 3 months after childbirth in the United States. ${ }^{(5)}$ In 21 low- and middle-income countries, the polled one-year contraceptive use rate for modern methods was $41.2 \%$. ${ }^{(6)}$ Studies in Shanghai showed that approximately $70 \%$ of migrant women in a suburban district and $88 \%$ of those living in downtown used contraceptives in the first 12 months postpartum. ${ }^{(7,8)}$ Kang et al. found that only $66 \%$ of postpartum women in rural areas of Hunan Province adopted contraceptive methods. ${ }^{(9)}$ We did not uncover any reports on overall postpartum contraceptive use nationwide in China.

Globally, an estimated 99 million unintended pregnancies occurred annually between 2010 and 2014. Of them, $56 \%$ ended in induced abortion. ${ }^{(10)}$ Ahrens et al. estimated that approximately $36 \%$ of pregnancies conceived within 18 months postpartum in the United States were unintended. ${ }^{(11)}$ Moore et al. showed that birth-to-pregnancy intervals in 50\% or more of pregnancies in 21 low- and middle-income countries were less than 23 months. ${ }^{(12)}$ Xiao et al. demonstrated that $10 \%$ of women of reproductive age in Enshi, Hubei province of China, experienced unintended pregnancies during the first year after delivery. ${ }^{(13)}$ None of them reported the prevalence of abortion during postpartum period.

Approximately 10 million abortions (9.6-9.9 million in 2016-2018) are performed each year in China. ${ }^{(14)}$ Reducing the number of abortions has been recognised by Chinese health authorities as one of the major approaches to improving women's reproductive health, and the health administrators from both central and local governments and a large number of family planning service providers have realised the importance of PPFP services for postpartum women. However, our knowledge of postpartum contraception, unintended pregnancy, and abortion across China is still very limited. We therefore conducted the present retrospective cohort study with the primary aim of exploring the prevalence of unintended pregnancy in the extended postpartum period (12-24 months after childbirth) in eastern, central, and western regions of China. Our secondary aims included the investigation of the prevalence of contraception and abortions during postpartum period. This information is essential for designing appropriate interventional PPFP strategies that will contribute to the reduction of unintended pregnancies and induced abortions in China overall. 


\section{Methods}

\section{Study sites and participant selection procedures}

We conducted an institutionally based retrospective cohort study between July 2017 and January 2018 at 60 public hospitals in China. Study participants were women who had given birth between 12 and 24 months earlier. First, we divided the country into three macrogeographic regions: eastern, central, and western. This is because there are huge socioeconomic differences and landscape variations among the three regions: Eastern China is the most developed and located near the eastern coastline of the country. Western China is the least developed and dominated by towering massifs and rolling plateaus. Central China is largely an agricultural area and generally less developed than the eastern coastal provinces but more developed than the western provinces. Second, we selected five provinces in each region (see Supplementary material, Figure S1), and appointed a senior physician or researcher as the provincial coordinator for each province. Third, we asked the provincial coordinators to provide a list of 10 hospitals with at least two each level-2 and level-3 general hospitals and two each level-2 and 2 level-3 Maternal and Child Hospitals, and this defined a sampling frame. A statistician randomly selected four hospitals from each province using computer-generated random numbers, randomly selecting a month between July 2015 and June 2016; and allocated it to each participating hospital as the initial month of investigation. The first 300 women who delivered a live birth in the selected month were eligible for this study. If the number of deliveries in the given month was less than 300 , women who delivered in the following month were invited for interviews.

\section{Sample size calculation}

The sample size was calculated based on a prevalence $(\mathrm{P})$ of unintended pregnancy of $8 \%$ among postpartum women in a given region. With a 0.01 uncertainty level (d), 95\% confidential interval (CI), and $5 \%$ margin of error, and using the formula $\mathrm{N}=Z^{2}{ }_{0.05} \mathrm{P}(1-\mathrm{P}) / \mathrm{d}^{2}$, we calculated a required sample size of 2828 . As we used a cluster (hospital) sampling technique to select the participants (as proposed by Donner, Birkett, and Buck), the sample size calculated assumed that individual randomization could be inflated by a design effect (DE) to reach the required level of statistical power under cluster randomization: $\mathrm{DE}=1+(\mathrm{n}-1) \rho$, where $\mathrm{n}$ was the number of individuals per cluster and $\rho$ was the intracluster correlation coefficient (ICC). ${ }^{(15)}$ When the primary outcome variable is a rate, the coefficient of variation in the outcome can be used as an alternative measure to the ICC, and is denoted by $x^{(16)}$ ( $x$ is usually derived from previous studies). However, we did not find any relevant studies where investigators reported the value of $x$. Based on experience, then, we set $\mathrm{DE}=2$, assuming a $x$ of 0.003 (using data from this study, we derived a $x$ of 0.001 ). We thus obtained a sample size of 5656 for each geographic region, or 283 participants for each hospital. We decided to recruit 300 participants at each hospital, which allowed for a $6 \%$ non-response rate.

\section{Data collection}

Data were collected with a structured questionnaire via face-to-face or telephone interviews. Two junior doctors and/or nurses selected as data collectors at each hospital were trained on the data collection procedures and the interview techniques. Recorded information included participants' background characteristics, pregnancy history, immediate postpartum contraceptive use, resumption of sexual activity, return of menstrual cyclicity, and occurrence and time of subsequent pregnancy and/or induced abortion after childbirth. All of the participants were interviewed between August 2017 and January 2018.

\section{Data processing and analysis}

We defined long-acting reversible contraceptives (LARCs) as intrauterine devices (IUDs) and implants. Modern contraceptive methods were defined as using one or more of the following method(s): male and female sterilizations, male and female condoms, combined oral pills (COCs), injectables, implants, IUDs, emergency 
contraceptive pills (ECPs), and spermicides. Traditional methods were defined as the use of the lactational amenorrhea method (LAM), rhythm/calendar method, and withdrawal. We defined unmet need for postpartum contraception as women who resumed sexual intercourse after delivery but did not use any contraceptive methods or only used traditional methods.

Data were entered into EPI INFO version 3.1, and we used SAS 9.4 (SAS Institute Inc.) for data analysis. Frequencies, percentages, means $+/-\mathrm{SD}$, and cross-tabulations were used to summarize descriptive statistics. Life-table analyses were used to calculate cumulative rates of contraceptive use, unintended pregnancy, and induced abortion during the first 24 months postpartum. We used the log-rank test to compare trends and Chi-squared test to compare rate differences between/among regions.

\section{Ethical considerations}

Ethical approval was obtained from the Institutional Review Board of Shanghai Institute of Planned Parenthood Research. Written (face-to-face interviews) and verbal (telephone interviews) consent was obtained from all the study participants, and they were assured that the information would remain confidential.

\section{Results}

\section{Background characteristics of study participants}

A total of 18,045 women who delivered a live birth between 12 and 24 months immediately preceding were interviewed for the present study. Of these, 6005, 6010, and 6030 were from the eastern, central, and western regions of China, respectively. Participants ranged in age from 16 to 50 years, with a mean age of $29.9 \pm$ 4.6 years. More young mothers (aged 20-24) were interviewed in the western (15\%) region relative to the eastern $(9 \%)$ and central $(7 \%)$ regions. Less than half $(48 \%)$ of the participants possessed an undergraduate education, about $22 \%$ had completed high school, and $22 \%$ had finished middle school. There were more less-educated women (middle school or below) in the western (33\%) region than in the eastern (23\%) or central (18\%) regions. Han nationality accounted for $88 \%$ of the study participants, although the proportion of minorities was significantly higher in the western $(12 \%)$ region relative to the eastern and central regions (5\%). Nearly half $(48 \%)$ of all participants were interviewed during the 13-18 months postpartum; this proportion was higher in the eastern (52\%) region compared with the central (44\%) and western (48\%) regions. Baseline demographics and characteristics of participants are shown in supplementary Table S1.

Contraceptive rates, unmet need, and modifications in contraceptive method use during the first 24 months postpartum

The cumulative 6-,12-, and 24-month contraception rates for participants using any method were $73.9 \%$ (95\% CI 73.3-74.5), 85.7\% (95\% CI 85.2-86.2), and 86.8\% (95\% CI 86.3-87.3), respectively; while the rates for modern methods were $62.7 \%$ (95\% CI $62.0-63.4$ ), $72.4 \%$ (95\% CI 71.8-73.1), and $73.2 \%$ (95\% CI 72.6 73.9), respectively. Among women who resumed sexual intercourse after childbirth, the 6-, 12-, and 24-month values for unmet need for postpartum contraception were $28.7 \%$ (95\% CI 28.0-29.4), $25.6 \%$ (95\% CI 25.026.2 ) and $23.8 \%$ (95\% CI 23.2-24.4), respectively. The level of contraceptive use was highest in the west (6-24 months: $77.8-88.6 \%$ ), followed by the east (6-24 months: 72.6-87.7\%), and lowest in the central region (6-24 months: 71.4-84.0\%) during the 24 months postpartum. In contrast, the percentage of unmet need for postpartum contraception was highest in the central region (6-24 months: 30.3-25.5\%) and lowest in the east (6-24 months: 27.2-22.4\%). (see Supplementary material, Table S2).

Condoms were dominant postpartum contraceptive method initially used after childbirth, accounting for approximately $2 / 3$ of study participants, followed by traditional methods (14\%), IUDs (2\%), and hormonal methods (1\%). More than 10\% (12\%) of participants did not use any methods. The variety of methods for first choice showed a similar pattern betweens regions. (see Supplementary material, Table S3). 
A considerable number of initial contraceptive users changed their first choices to current methods. Of the 12,097 initial condom users, $10 \%$ switched to IUDs, $2 \%$ used traditional methods, and $4 \%$ became non-users; approximately $46 \%$ of traditional-method users converted to condoms, $13 \%$ changed to IUDs, and $8 \%$ became non-users; $7 \%$ of users replaced IUDs with condoms, $4 \%$ removed IUDs and became non-method users; $30 \%$ of combined oral contraceptive (COC) users changed to condoms, $12 \%$ turned to IUDs, and $3 \%$ to traditional methods. More than half (52\%) of emergency contraceptive users turned to condom use and 19\% to IUDs. (see Supplementary material, Table S4).

\title{
Pregnancy and unintended pregnancy during the first 24 months postpartum
}

Our study encompassed a total of 2186 pregnancies after index births. Of these, 1693 (77\%) were unintended. More than half $(52 \%, 1141)$ of the pregnancies were conceived within the first 12 months after delivery, and $83 \%(949 / 1141)$ of these were unintended.

Results of life-table analyses showed that the overall cumulative 6-, 12-, and 24-month pregnancy rates after childbirth were $1.7 \%, 6.3 \%$, and $18.5 \%$, respectively. The rates were $1.5 \%, 5.6 \%$, and $15.5 \%$ in the east; $1.1 \%$, $5.0 \%$, and $17.8 \%$ in the central region; and $2.3 \%, 8.4 \%$, and $21.8 \%$ in the west, respectively (Table 1 ). As shown in Figure 1, the curve for the western region increased faster than that for the other two regions. Our log-rank test results indicated that pregnancy rate was significantly higher in the west than in either of the other two regions during the first two years postpartum (all $P<0.001$ ), while the rates for the eastern and central regions were essentially similar $(P=0.329)$.

The overall cumulative 6-,12-, and 24-month unintended pregnancy rates were $1.4 \%, 5.3 \%$, and $13.6 \%$, respectively. The regional rates were $1.4 \%, 4.8 \%$, and $11.8 \%$ in the east; $1.0 \%, 4.2 \%$, and $13.3 \%$ in the central region; and $2.0 \%, 6.9 \%$, and 15.6 in the west, respectively (Table 1). The curve reflecting the unintended pregnancy rate rose faster in the west than in the other two regions during the 24 months postpartum (Figure 2, log-rank test, $P<0.001$ ), and as the lines for the eastern and central regions overlapped, we observed no statistical difference in the rates between the latter two regions during the 24 months after delivery (log-rank test, $P=0.183)$.

\section{Abortion during the first 24 months postpartum}

Of the 1141 pregnancies conceived within 12 months of a delivery, $708(62 \%)$ ended in abortion, accounting for $75 \%$ (708/949) of unintended pregnancies during the postpartum period. However, only 8.3\% (16/192) of the pregnancies not reported as unintended pregnancies ended in abortion. By 24 months postpartum, $59 \%(1286 / 2186)$ of all pregnancies and $76 \%$ (1286/1693) of unintended pregnancies ended in abortion. Only $10 \%(50 / 493)$ of the non-unintended pregnancies were terminated by abortive procedures.

Results of life-table analyses showed that the overall cumulative 6-, 12-, and 24-month rates of induced abortion were $1.1 \%, 4.0 \%$, and $10.8 \%$, respectively (Table 1 ). In addition, the cumulative $6-$, $12-$, and 24 month unintended pregnancy rates were $1.0 \%, 4.0 \%$, and $10.2 \%$ in the east; $0.7 \%, 3.0 \%$, and $10.0 \%$ in the central region; and $1.4 \%, 5.1 \%$, and $12.1 \%$ in the west, respectively.

The cumulative rate for induced abortions increased fastest in the west and slowest in the central region during the 24 months postpartum (Figure 3). Women in the west were significantly more likely to undergo an abortion relative to their counterparts in the eastern $(P=0.008)$ and central regions $(P<0.001)$, and women in the east were significantly more likely to have a pregnancy terminated by abortion compared to those in the west $(P=0.003)$ during the first two years after childbirth.

\section{Discussion}

\author{
Main findings
}


We report, for the first time, the prevalence of contraceptive use, unintended pregnancy, and induced abortion during 24 months postpartum in eastern, central, and western regions of China and in China overall. The prevalence of postpartum contraceptive use is high but dominated by condoms and traditional methods. The variety of methods for first choice showed a similar pattern betweens regions. Approximately 1 in 7 women experienced unintended pregnancy, and 1 in 10 underwent induced abortion during 24 months postpartum. The high risks of unintended pregnancy and induced abortion during the extended postpartum period can be mainly attributed to overreliance on the less-effective contraceptive methods and high level of unmet need for postpartum contraception across the country.

\section{Strengths and limitations}

This is the first large-scale, multi-province, retrospective cohort study that investigated prevalence of postpartum contraception, unintended pregnancy and induced abortion nationwide in China. The study manifests several limitations. Our study relied on women's retrospective reporting of their postpartum contraception, pregnancy, and abortion; and hence may be subject to recall bias. We recorded the timing with respect to initiation of postpartum contraception, resumption of sexual intercourse, pregnancy, and abortion by rounding the number of months; and this may not have provided adequate accuracy in measuring cumulative rates.

\section{Interpretation}

\section{Postpartum contraception}

The present study revealed that between $86 \%$ and $87 \%$ of postpartum women used at least one contraceptive method during the first 12 and 24 months after childbirth, respectively. Our findings were similar to that of a US study, in which, $88 \%$ of postpartum women used contraceptive methods in 12 states and New York City. ${ }^{(17)}$ In our study, $73 \%$ of contraceptive initiators adopted modern methods during the first 24 months postpartum, which was similar to the finding of the 2006-2010 National Survey of Family Growth in the United States (76\% at 18 months postpartum). ${ }^{(5)}$ However, our estimate was higher than the results from most low- and middle-income countries - e.g., $10 \%$ in Ethiopia, $25 \%$ in Niger and Ghana, $34 \%$ in India, ${ }^{(6)}$ and $65-70 \%$ in Indonesia. ${ }^{(18)}$

Unexpectedly, we found that by 12 months postpartum, $26 \%$ of respondents who had resumed sex had an unmet need for contraception. Previous studies showed that the levels of unmet need for postpartum contraception were very high in most low- and middle-income countries; e.g., $33 \%-38 \%$ in Indonesia, ${ }^{(18)} 52 \%$ in Nepal, ${ }^{(19)} 32 \%$ in India, $46 \%$ in Kenya, $59 \%$ in Nigeria, and $97 \%$ in Pakistan. ${ }^{(6)}$ It is important to note that the definition of "unmet need" varied among studies. We defined unmet need for postpartum contraception as women who resumed sex after delivery, but did not use any contraceptive methods or used traditional methods. Dev et al. in a systematic review defined unmet need for postpartum contraception based upon the data available, e.g., as women who did not want a child in the subsequent two years but also were not using modern contraceptives. ${ }^{(6)}$ Contraceptive methods are free for married couples in China, and unmarried couples can also readily receive free short-acting methods from family planning service points. Hence, the high level of unmet need for postpartum contraception may largely reflect inadequate PPFP services during the process of prenatal and postnatal care in China.

Condoms accounted for $79 \%$ of the first postpartum contraceptive choices; followed by traditional methods (16\%). IUDs accounted for only $3 \%$, and no participants underwent sterilisation or used contraceptive implants. This specific mix of contraceptive methods was very different from findings reported elsewhere. For example, $28 \%$ of US women used hormonal contraceptives, $11 \%$ underwent female sterilisation, $2 \%$ of male partners underwent sterilisation, $6 \%$ used LARC methods, and $25 \%$ used less effective methods (including condoms, spermicides, and traditional methods) at 3 months postpartum. ${ }^{(5)}$ Using this classification, only $3 \%$ of postpartum women initiated the use of highly effective contraceptive methods in our study. Although a substantial number of short-acting method users switched to IUDs during the extended postpartum periodmaking IUDs (14\%) the second commonly used contraceptive methods at the time of interview-condom use predominated (75\%). According to a WHO document, when used in typical fashion, the effectiveness 
of condoms, withdrawal, and periodic abstinence is about $87 \%, 80 \%$, and $77 \%$, respectively -all far below that for LARCs (IUDs, 99\%; implants, 99.9\%). ${ }^{(20)}$ The mixed use pattern of postpartum methods therefore raises concerns of an elevated risk of unintended pregnancy and induced abortion among postpartum Chinese women.

\section{Unintended pregnancy and induced abortion}

The overall 12-month rates of pregnancy, unintended pregnancy, and induced abortion are $6.3 \%, 5.3 \%$, and $4.0 \%$, respectively; indicating that $84 \%$ of all pregnancies conceived during the first 12 months postpartum were unintended, and $75 \%$ (accounting for $63 \%$ of all pregnancies) were terminated by abortion procedures. By 24 months postpartum, the cumulative rates of pregnancy, unintended pregnancy, and induced abortion increased to $18.5 \%, 13.6 \%$, and $10.8 \%$, respectively; $77 \%$ of pregnancies were unintended; and $76 \%$ of unintended pregnancies ended in abortion. Intriguingly, several local studies reported even higher risks of unintended pregnancy. For instance, investigators using a survey in Enshi, Hubei Province, estimated that $10 \%$ of women underwent an unintended pregnancy during the first 12 months postpartum. ${ }^{(13)}$ Ruo et al. estimated that $9 \%$ of postpartum women had an unintended pregnancy within 12 months after childbirth in Pengshan District, Sichuan Province. ${ }^{(21)}$ According to an official report, a total of 17.23 million babies were delivered in China in 2017. ${ }^{(22)}$ If we posited that these babies were all singleton births, based on our estimates there would be approximately 1 million pregnancies conceived within 12 months postpartum in women who gave a birth in 2017. Of these pregnancies, 913,000 were unplanned and 689,000 would have ended in abortion. The direct medical expenses for abortion services (1500 Yuan per abortion surgery) are approximately 1 billion Yuan (about 14 million USD). These results demonstrated that the first 12 months after childbirth constituted an essential period for women to take contraceptives so as to prevent an unintended pregnancy and induced abortion in China. Our findings indicated an urgent need for revising the current national PPFP program with clearer guidance on counselling postpartum women regarding LARC methods in China.

\section{Variety between regions}

We found that slightly more women from western China adopted contraception - in particular IUDs - than their eastern and central counterparts. These results may be due in part to more participants having two or more children in the western region than in the eastern and central regions. The desire for limiting births may also be stronger for women with more children. Somewhat surprisingly, the cumulative rates of unintended pregnancy and abortion were significantly higher in the western region relative to eastern and central regions. One possible reason for this is that the time of contraceptive initiation may be later for women in the western region compared with counterparts in eastern and central regions of China. However, further studies are needed to elucidate the differences in contraceptive behaviours and subsequent pregnancy among women living in various geographic regions of China.

\section{Conclusion}

The prevalence of postpartum contraception is relatively high in China but dominated by less effective methods. This finding suggests that the overall quality of PPFP services in China is not optimal as the prevalence of unintended pregnancy and induced abortion is higher during the 24 months postpartum. A national PPFP program that exhibits a clear guide on how to deliver high-quality PPFP to postpartum women is needed in China. Service providers should work on counselling postpartum women and their partners with respect to LARC methods, and to effectively and reliably use short-acting contraceptive methods during the postpartum period.

\section{Disclosure of Conflicts}

The authors declare no competing interests.

\section{Contribution to authorship}

YC conceived and designed the study, obtained funding for the study, led the execution of the study including 
data analysis and interpretation, drafted the first draft of the manuscript and critically reviewed final drafts. YL assisted in designing, executing, interpreting the data and critically revised the draft of the manuscript. XG, FJ, YZ, XH, LJ, TC, XD, XH, YL, WL, GQ, JS, WX, LZ, and YZ conducted data collection and critically revised the draft of the manuscript. YZ contributed to data analysis, interpretation and review of the manuscript. All authors read and approved the final manuscript.

\section{Details of ethics approval}

This study was approved by Shanghai Institute of Planned Parenthood Research in 2017 (PJ2017-17). All participants provided informed consent.

\section{Funding}

This work was supported by Science and Technology Climbing Funds from the Shanghai Institute of Planned Parenthood Research (grant no. PD2017-10)

\section{Acknowledgements}

We gratefully acknowledge the contributions of investigators from the 60 participating hospitals with respect to data collection and data entry.

\section{References}

1. DaVanzo J, Hale L, Razzaque A, Rahman M. Effects of interpregnancy interval and outcome of the preceding pregnancy on pregnancy outcomes in Matlab, Bangladesh. BJOG. 2007;114(9):1079-87.

2. Conde-Agudelo A, Rosas-Bermudez A, Castano F, Norton MH. Effects of birth spacing on maternal, perinatal, infant, and child health: a systematic review of causal mechanisms. Stud Fam Plann. 2012;43(2):93114.

3. Cleland J, Bernstein S, Ezeh A, Faundes A, Glasier A, Innis J. Family planning: the unfinished agenda. Lancet. 2006;368(9549):1810-27.

4. World Health Organization, USAID, Mathernal and Child Health Integrated Program. Programming Strategies for Postpartum Family Planning. Geneva: World Health Organization; 2013.

5. White K, Teal SB, Potter JE. Contraception after delivery and short interpregnancy intervals among women in the United States. Obstet Gynecol. 2015;125(6):1471-7.

6. Dev R, Kohler P, Feder M, Unger JA, Woods NF, Drake AL. A systematic review and meta-analysis of postpartum contraceptive use among women in low- and middle-income countries. Reprod Health. 2019;16(1):154.

7. Huang YM, Merkatz R, Kang JZ, Roberts K, Hu XY, Di Donato F, et al. Postpartum unintended pregnancy and contraception practice among rural-to-urban migrant women in Shanghai. Contraception. 2012;86(6):731-8.

8. Huang YM, Kang J, Hu X, Chen R, Cheng L. Study on unintended pregnancy and risk factors among postnatal women in Shanghai. Reproduction and Contraception. 2008;28(5):286-90.

9. Kang C, Li P, Liu X, Ding Y, Wang X, Zhou H. Use of contraceptives and uptake of long-acting reversible contraception among postpartum women in rural China. BMJ sexual \& reproductive health. 2018.

10. Singh S, Reme L, Sedg G, Kwok L, Onda T. Abortion worldwide 2017:uneven progress and uneuqal access. New York: Guttmacher Institute; 2018.

11. Ahrens KA, Thoma ME, Copen CE, Frederiksen BN, Decker EJ, Moskosky S. Unintended pregnancy and interpregnancy interval by maternal age, National Survey of Family Growth. Contraception. 2018;98(1):52-5.

12. Moore Z, Pfitzer A, Gubin R, Charurat E, Elliott L, Croft T. Missed opportunities for family planning: an analysis of pregnancy risk and contraceptive method use among postpartum women in 21 low- and middle-income countries. Contraception. 2015;92(1):31-9. 
13. Xiao H, Qin $\mathrm{C}$, Yan $\mathrm{H}, \mathrm{Li} \mathrm{S}, \mathrm{Xu}$ D. Prevalence and related reasons of unintended pregnancy in the first year of delivery among married women in Enshi. Chinese Journal of Disease Control \& Prevention. 2014;18(9):876-9.

14. National Health Commission. China's Health Statistics Yearbook 2018. Beijing: Peking union medical college press; 2019.

15. Donner A, Birkett N, Buck C. Randomization by cluster. Sample size requirements and analysis. American journal of epidemiology. 1981;114(6):906-14.

16. Hayes RJ, Bennett S. Simple sample size calculation for cluster-randomized trials. Int J Epidemiol. 1999;28(2):319-26.

17. Whiteman M, Curtis K, Hillis S, Zapata L, D'Angelo D, SL Farr, et al. Contraceptive Use Among Postpartum Women - 12 States and New York City, 2004-2006. MMWR weekly. 2009;58(30):821-6.

18. Wilopo SA, Setyawan A, Pinandari AW, Prihyugiarto T, Juliaan F, Magnani RJ. Levels, trends and correlates of unmet need for family planning among postpartum women in Indonesia: 2007-2015. BMC Womens Health. 2017;17(1):120.

19. Mehata S, Paudel YR, RanjuMehta, Dariang M, Poudel P, Barnett4 S. Unmet Need for Family Planning in Nepal during the First Two Years Postpartum. BioMed Research International [Internet]. 2014; 2014:[1-9 pp.].

20. (WHO/RHR); WHODoRHaR, (CCP); JHBSoPHCfCP, Project. KfH. Family Planning: A Global Handbook for Providers (2018 update). Baltimore and Geneva: CCP and WHO; 2018.

21. Ruo L, Zhang J, Luo J. An investigation of the unintended pregnancy and induced abortion in Pengshan District of Meishai City. Journal of Hainan Medicine. 2018;29(9):1305-8.

22. National Bureau of Statistics. In 2017, the State economic operation was stable, positive and better than expected: National Bureau of Statistics,; 2018 [cited 2020 June 6]. Available from: http://www.stats.gov.cn/tjsj/zxfb/201801/t20180118_1574917.html.

Table 1. Cumulative 6-, 12-, and 24-month rates of pregnancy, unintended pregnancy and induced abortion, by geographic regions

\begin{tabular}{|c|c|c|c|c|c|c|c|c|c|}
\hline $\begin{array}{l}\text { Months } \\
\text { since } \\
\text { delivery }\end{array}$ & Overall & Overall & Eastern & refgiasthern & region & Central & regientral & region & Western \\
\hline & $\%$ & $95 \%$ & $\%$ & $95 \%$ & & $\%$ & $95 \%$ & & $\%$ \\
\hline & & CI & & CI & & & CI & & \\
\hline \multicolumn{10}{|c|}{$\begin{array}{l}\text { All } \\
\text { pregnancy }\end{array}$} \\
\hline 6 & 1.7 & $1.5-1.8$ & 1.5 & $1.2-1.9$ & & 1.1 & $0.8-1.3$ & & 2.3 \\
\hline 12 & 6.3 & $6.0-6.7$ & 5.6 & $5.0-6.2$ & & 5.0 & $4.4-5.5$ & & 8.4 \\
\hline 24 & 18.5 & $\begin{array}{l}16.8- \\
20.1\end{array}$ & 15.5 & $\begin{array}{l}12.6- \\
18.3\end{array}$ & & 17.8 & $\begin{array}{l}15.2- \\
20.4\end{array}$ & & 21.8 \\
\hline \multicolumn{10}{|c|}{$\begin{array}{l}\text { UnintendedUnintendedUnintended } \\
\text { pregnancy pregnancy pregnancy }\end{array}$} \\
\hline 6 & 1.4 & $1.3-1.6$ & 1.4 & $1.1-1.7$ & & 1.0 & $0.7-1.2$ & & 2 \\
\hline 12 & 5.3 & $5.0-5.6$ & 4.8 & $4.3-5.4$ & & 4.2 & $3.6-4.7$ & & 6.9 \\
\hline 24 & 13.6 & $\begin{array}{l}12.2- \\
15.0\end{array}$ & 11.8 & $9.3-14.4$ & & 13.3 & $\begin{array}{l}10.9- \\
15.6\end{array}$ & & 15.6 \\
\hline $\begin{array}{l}\text { Induced } \\
\text { abortion }\end{array}$ & $\begin{array}{l}\text { Induced } \\
\text { abortion }\end{array}$ & & & & & & & & \\
\hline
\end{tabular}




\begin{tabular}{|c|c|c|c|c|c|c|c|}
\hline $\begin{array}{l}\text { Months } \\
\text { since } \\
\text { delivery }\end{array}$ & Overall & Overall & Eastern & regiashern region & Central & regientral region & Westerr \\
\hline 6 & 1.1 & $0.9-1.2$ & 1.0 & $0.8-1.3$ & 0.7 & $0.5-1.0$ & 1.4 \\
\hline 12 & 4.0 & $3.7-4.3$ & 4.0 & $3.5-4.5$ & 3.0 & $2.6-3.4$ & 5.1 \\
\hline 24 & 10.8 & $9.5-12.1$ & 10.2 & $7.8-12.7$ & 10.0 & $7.0-12.1$ & 12.1 \\
\hline
\end{tabular}

\section{Hosted file}

Figure1.docx available at https://authorea.com/users/351824/articles/476302-contraceptionpregnancy-and-induced-abortion-within-24-months-of-delivery-in-eastern-central-andwestern-regions-of-china-a-retrospective-cohort-study

\section{Hosted file}

Figure2.docx available at https://authorea.com/users/351824/articles/476302-contraceptionpregnancy-and-induced-abortion-within-24-months-of-delivery-in-eastern-central-andwestern-regions-of-china-a-retrospective-cohort-study

\section{Hosted file}

Figure3.docx available at https://authorea.com/users/351824/articles/476302-contraceptionpregnancy-and-induced-abortion-within-24-months-of-delivery-in-eastern-central-andwestern-regions-of-china-a-retrospective-cohort-study 